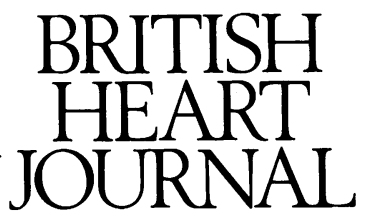

Editorial

\title{
Power spectrum analysis of heart rate variability: a tool to explore neural regulatory mechanisms
}

Even at rest the RR interval changes continuously around its mean value. Neural mechanisms account for part of this variability. Conventionally the interplay between sympathetic and vagal modulation of sinus node pacemaker activity is believed to be reciprocal-with increased activity in one system being accompanied by decreased activity in the other. Important new information has been generated by coupling the analysis of heart rate variability (HRV) to the concept of sympatho-vagal balance. ${ }^{1}$

\section{Assessment of heart rate variability \\ THE TIME DOMAIN}

Initial measurements of HRV were based on simple measures of $R R$ interval variation in the time domain. Despite their simplicity, these measurements, the most common of which is standard deviation (SD), proved to be useful clinical tools. Murray et al found that the SD was reduced in diabetic patients without the usual cardiovascular signs of autonomic neuropathy, ${ }^{2}$ and the large cooperative study of Kleiger and coworkers showed that SD predicted outcome in patients who had survived an acute myocardial infarction. ${ }^{3}$ Murray et al and Kleiger et al interpreted the reduction in HRV as a sign of decreased parasympathetic activity. Furthermore, Kleiger et $\mathrm{al}^{3}$ interpreted a reduction in SD as a sign of increased sympathetic activity too. Hence SD was implicitly proposed as a simple tool to assess the sympath-ovagal balance. The SD provides an evaluation of overall HRV but as a measure of changes in sympatho-vagal balance it is limited.

\section{THE FREQUENCY DOMAIN}

Various algorithms can be used to assess the number, centre frequency, and amplitude of the oscillatory components hidden in the variability signal. ${ }^{4}$ Most studies have relied either on fast Fourier transformation (FFT) ${ }^{56}$ or on the autoregressive approach (AR).$^{78} \mathrm{FFT}$ is easier to implement but requires a priori selection of the number and frequency range of the bands of interest, whereas AR algorithms automatically give the number, centre frequency, and associated power of oscillatory components without the need for a priori decisions. Both approaches assume stationary conditions that strictly speaking are unknown in biological systems. Thus a practical compromise has to be made between the length of the event series and the theoretical mathematical requirements. In this sense, a further advantage of the AR algorithms is that short segments of data provide an efficient spectral estimation (for example 200 cycles rather than the more usual 512 cycles) which are more likely to be stationary and easier to obtain during clinical studies.

During the past decade three major components of the HRV spectrum have been recognised-with frequencies centred around $0.0 \mathrm{~Hz}$ (VLF, very low frequency), $0 \cdot 10$ $\mathrm{Hz}$ (LF, low frequency), and $0.25 \mathrm{~Hz}$ (HF, high frequency) (figure). Similar frequencies characterise the spectrum of variability in arterial pressure but only the LF and HF components of the two variability signals show a statistically significant association. This accords with the interpretation that in the circulatory closed-loop system the LF oscillation corresponds to vasomotor activity and the HF oscillation corresponds to respiratory activity. This makes obsolete the initial approach of dividing the frequency range $0.02-0.5 \mathrm{~Hz}$ into three predetermined bands. ${ }^{6}$ The power of the LF and HF spectral components of HRV can be expressed as absolute $\left(\mathrm{ms}^{2}\right)$ or normalised units $(0-100)$. The normalisation procedure $^{8}$ has proved crucial to the interpretation of data.

In physiological conditions the sympathetic excitation that leads to tachycardia is accompanied by a reduction
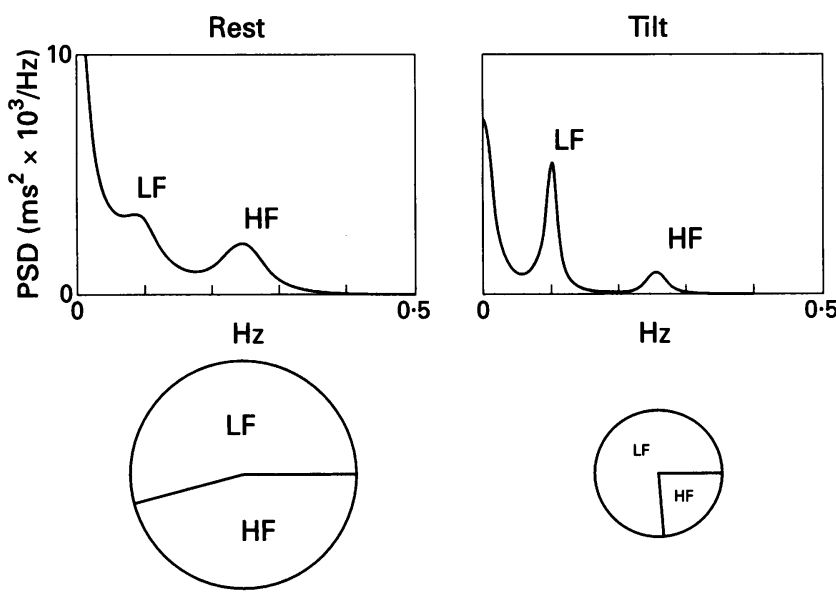

Total variance $2000 \mathrm{~ms}^{2}$ $L F=573 \mathrm{~ms}^{2}=52.84 \mathrm{nu}$

Total variance $633 \mathrm{~ms}^{2}$ $\mathrm{LF}=303 \mathrm{~ms}^{2} \equiv 74.34 \mathrm{nu}$ $\mathrm{HF}=93 \mathrm{~ms}^{2}=22 \cdot 85 \mathrm{nu}$

Example of spectral analysis of $R R$ interval variability in a healthy subject examined at rest and during a passive tilt. At rest there are two major components (LF, low frequency and $H F$, high frequency) of similar power whereas during tilting the LF component predominates. In this case the total variance during tilting is markedly reduced so both $L F$ and $H F$ powers seem to be diminished when expressed in absolute units. The use of normalised units (nu) clearly indicates the altered relation between the two spectral components induced by tilting. The pie charts show this relative distribution together with the absolute power of the two components (represented by the area). 
in the total power and in the SD of HRV. During vagal activity the reverse is true. When absolute units are used these changes in total power are usually seen to influence both LF and HF components in the same direction: however, in several physiological and pathophysiological conditions the sympatho-vagal balance can shift without significant changes in heart period and total power. ${ }^{1}$ The sympatho-vagal balance seems to be accurately reflected by the fractional distribution of power across the frequency axis. This balance can be assessed by the relation between LF and HF components in normalised units or by the LF/HF ratio. This new method of analysis ${ }^{18}$ has led us to a reinterpretation of the biological significance of spectral components.

Since the initial studies ${ }^{5}$ the $\mathrm{HF}$ component has been regarded as a reliable marker of vagal activity. The interpretation of the LF component has been more controversial. Pomeranz et al regard the LF component as being mediated entirely by vagal activity in supine position and by both sympathetic and vagal activities in the standing position. ${ }^{9}$ They reported that in supine subjects under controlled respiration (and hence in a state of vagal predominance) atropine drastically reduced both the LF and HF components: however, they did not recognise that the remaining power was mainly distributed in the LF range. An alternative view, supported by our studies ${ }^{18}$ holds that LF provides a marker of sympathetic activity. So the findings of Pomeranz et $a l^{9}$ could be interpreted as reflecting the sympathetic predominance induced by atropine administration. Indeed, from the beginning of our investigation ${ }^{7}$ we found that in healthy individuals the LF component of both heart rate and arterial pressure variability was increased by tilt, mental stress, or moderate physical exercise. In animal experiments, the LF component was increased by moderate hypotension, physical activity, and occlusion of the coronary artery or common carotid arteries. In all these conditions, which are characterised by a shift of sympatho-vagal balance towards sympathetic predominance, the increase in the LF component of HRV was accompanied by a decrease in the HF component (both assessed in normalised units). ${ }^{1}$ The HF component of HRV was increased in humans by controlled respiration, cold stimulation of the face, and rotational stimuli-all conditions that increase vagal activity.

None the less, electrophysiological studies show that LF and HF oscillations are present in both the variability signals of impulse activity recorded simultaneously from the sympathetic and vagal efferent fibres that participate in cardiac innervation: thus in our hypothesis we do not restrict LF and HF components to any particular neural circuit. On the contrary, we propose that sympathetic and vagal activities interact, not only in modulating target functions but also in determining their rhythmic properties.

Spectrum analysis can also be carried out on Holter recordings of the electrocardiogram and the arterial pressure. ${ }^{10}$ In both instances the presence of artefacts, arrhythmias, and the quality of analogue recordings must be critically evaluated. Under appropriate conditions it is possible to assess the LF and HF components over the
24 hour period: in healthy individuals these components show continuous fast oscillations, a clear circadian rhythm, and reciprocal fluctuation (with a fall of LF and a rise of HF during the night). These changes, however, are more clearly appreciated when the values are expressed as normalised units: for example, because of a corresponding increase in total power the nocturnal fall of the LF component could appear as an unexpected increase if it were expressed in absolute units.

The biological relevance of spectral analysis of HRV is severely limited when variance is restricted, as it is in strenuous exercise or in diseases such as advanced heart failure or complicated myocardial infarction. Advanced heart failure or complicated myocardial infarction are likely to be accompanied by decreased responsiveness of the sinus node to neural modulation and therefore the extremely low variance would not reflect a simple increase in sympathetic activity. In diabetic autonomic neuropathy HRV can be considerably reduced, but the distribution of the residual variance results in an unmodified $\mathrm{LF} / \mathrm{HF}$ ratio suggesting a simultaneous effect on both sympathetic and vagal modulations.

When the signal produced by heart rate variability is adequate the relative powers of LF and HF components and their reciprocal relation seem to parallel the dynamic balance between sympathetic and vagal activities. Examination of these components will exemplify the sympatho-vagal balance under normal and abnormal conditions.

Centro Ricerche Cardiovascolari CNR,

ALBERTO MALLIANI FEDERICO LOMBARDI MASSIMO PAGANI

Medicina Interna II, Ospedale " $L$. Sacco", Universita di Milano

1 Malliani A, Pagani M, Lombardi F, Cerutti S. Cardiovascular neural regulation explored in the frequency domain. Circulation 1991;84:482-92. Murray A, Ewing DJ, Campbell IW, Neilson JMM, Clarke BF. RR interval variations in young male diabetics. Br Heart $\mathcal{f} 1975 ; 37: 882-5$.

3 Kleiger RE, Miller JP, Bigger JT, Moss AR. Multicenter Post-infarction Research Group. Decreased heart rate variability and its association with increased mortality after acute myocardial infarction. Am $\mathcal{f}$ Cardiol 1987;59:256-62.

4 Kay SM, Marple SL Jr. Spectrum analysis-A modern perspective. Proc IEEE 1981;69:1380-419.

5 Kitney RI, Rompelman O. The study of heart rate variability. Oxford: Clarendon Press, 1980

6 Akselrod S, Gordon D, Ubel FA, Shannon DC, Barger AC, Cohen RJ. Power spectrum analysis of heart rate fluctuations: A quantitative probe of beat-to-beat cardiovascular control. Science 1981;213:220-2.

7 Brovelli M, Baselli G, Cerutti S, Guzzetti S, Liberati D, Lombardi F, Malliani A, Pagani M, Pizzinelli P. Computerized analysis for an experimental validation of neurophysiological models of heart rate control. Comput Cardiol 1983;205-8.

8 Pagani M, Lombardi F, Guzzetti S, Rimoldi O, Furlan R, Pizzinelli P, Sandrone G, Malfatto G, Dell'orto S, Piccaluga E, Turiel M, Baselli G, Cerutti S, Malliani A. Power spectral analysis of heart rate and arterial pressure variabilities as a marker of sympatho-vagal interaction in man pressure variabilities as a marker of sympath
and conscious dog. Circ Res 1986;59:178-93.

9 Pomeranz B, Macaulay RJB, Caudill MA, Kutz I, Adam D, Gordon D, Kilborn B, Macaulay RJB, Caudil MA, Kutz I, Adam D, Gordon $\mathrm{D}$, Kilborn KM, Barger AC, Shannon DC, Cohen RJ, Benson $H_{\text {. }}$. Assessment of autonomic function in hum
analysis. Am $\mathcal{F}$ Physiol $1985 ; 248(\mathrm{H}): 151-3$.

10 Furlan R, Guzzetti S, Crivellaro W, Dassi S, Tinelli M, Baselli G, Cerutti S, Lombardi F, Pagani M, Malliani A. Continuous 24 hour assessment of the neural regulation of systemic arterial pressure and $R R$ variabilities in ambulant subjects. Circulation 1990;81:537-47. 